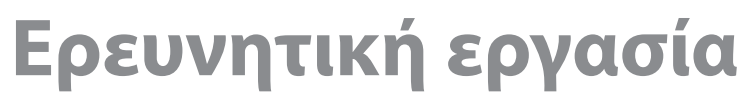 Research article
}

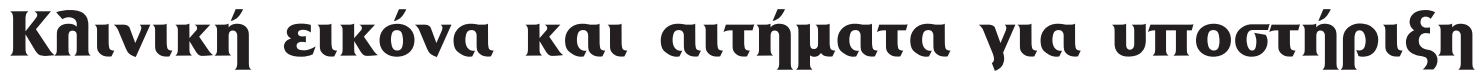

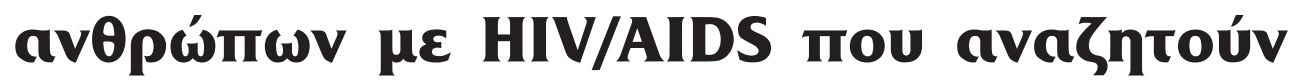

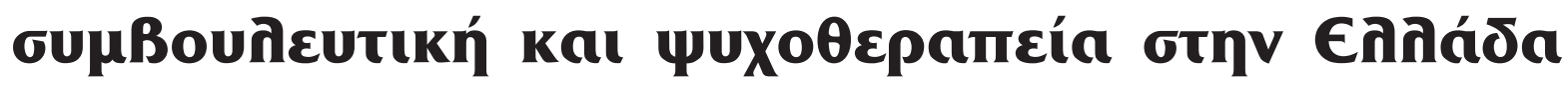

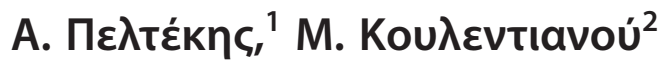

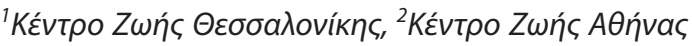

廿uxıатрıкń 2019, 30:120-128

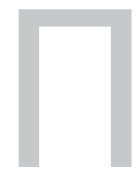

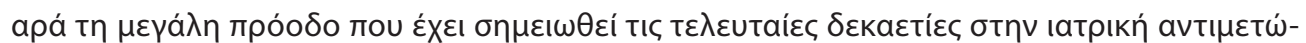

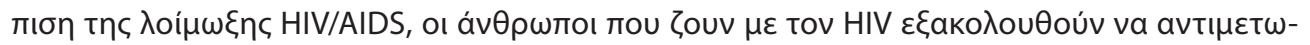

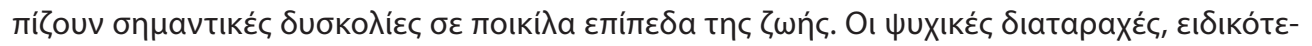

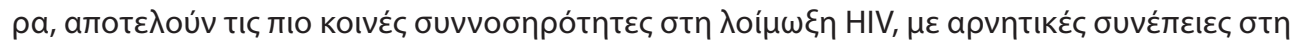

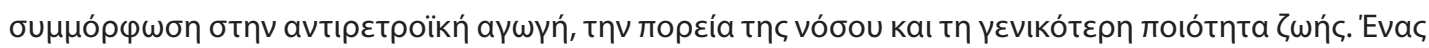

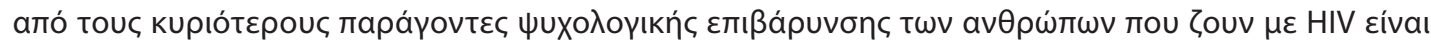

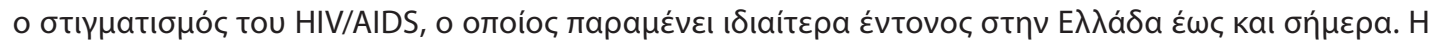

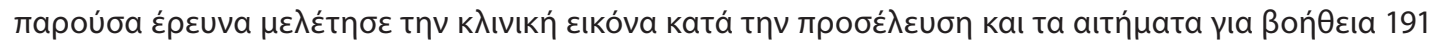

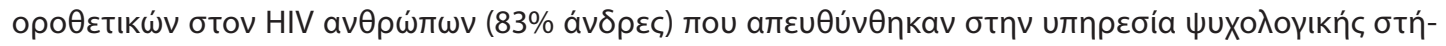

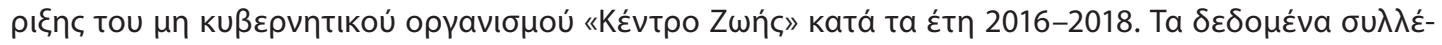

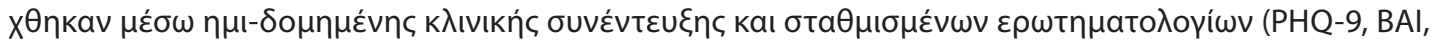

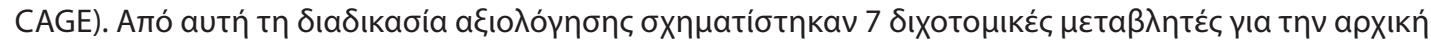

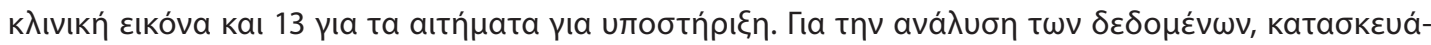

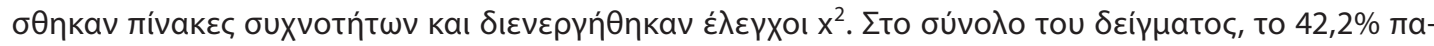

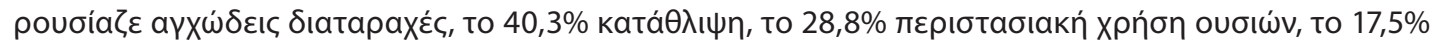

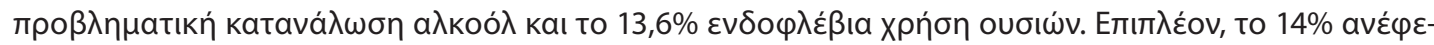

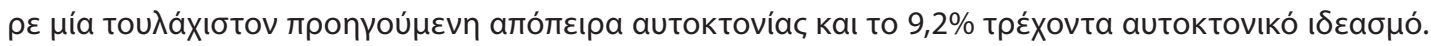

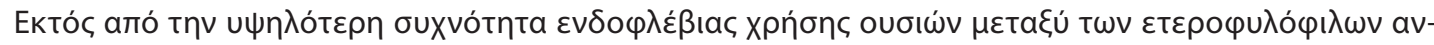

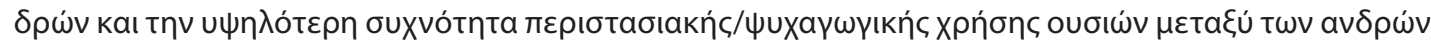

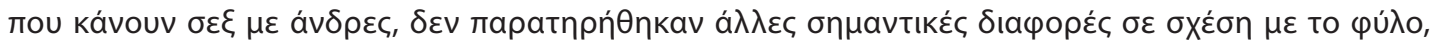

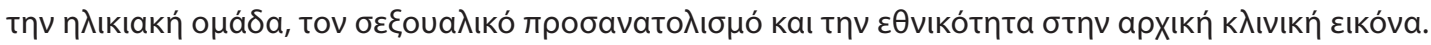

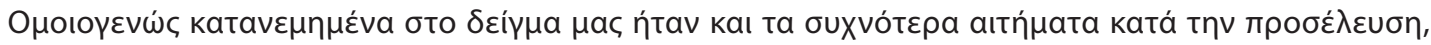




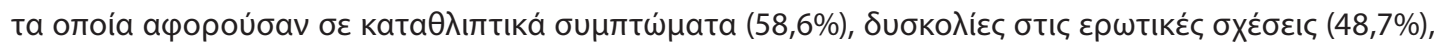

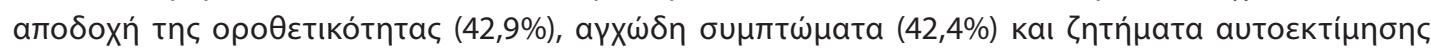

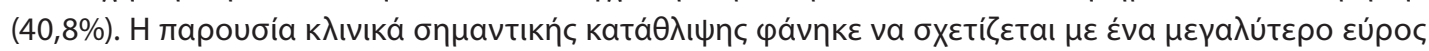

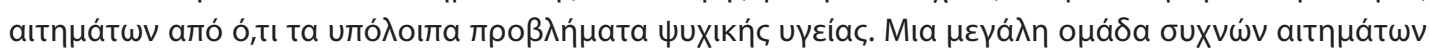

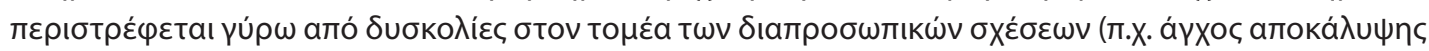

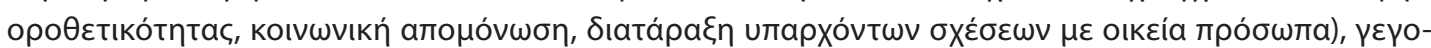

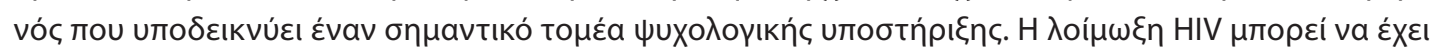

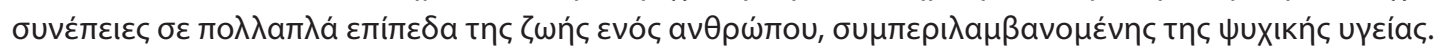

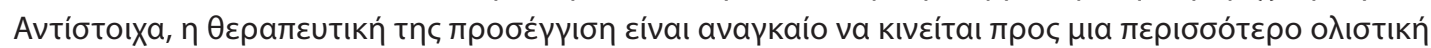

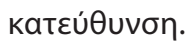

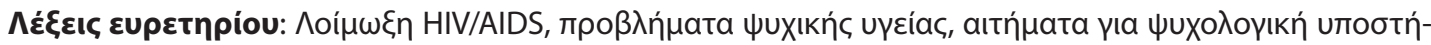

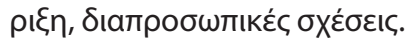

\section{Eıбaywyń}

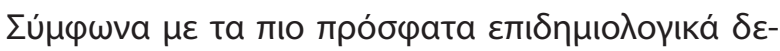

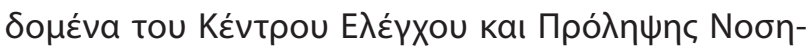

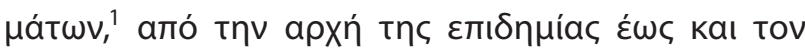

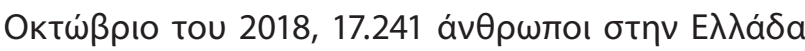

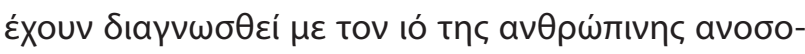

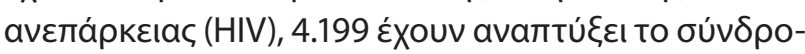

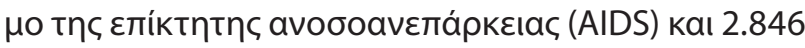

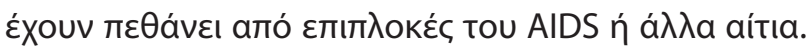

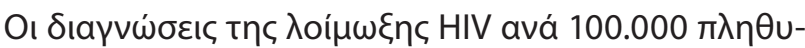

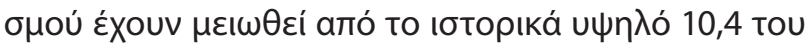

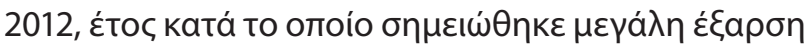

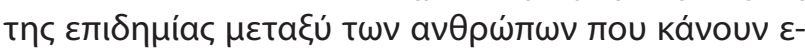

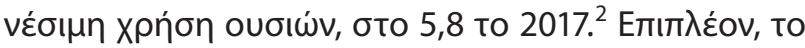

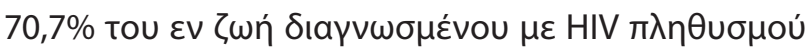

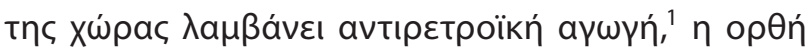

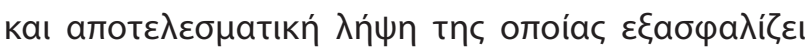

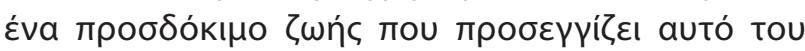

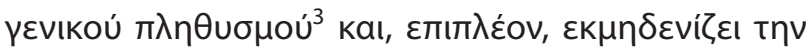

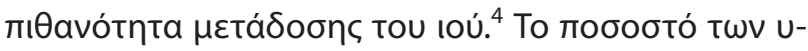

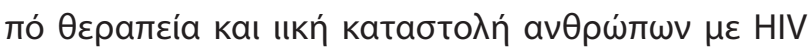

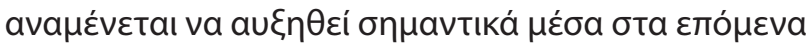

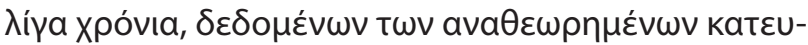

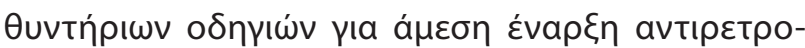

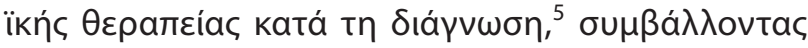

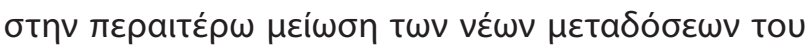

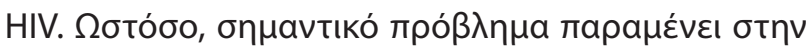

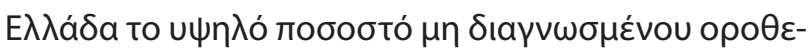

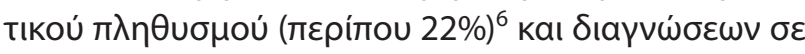

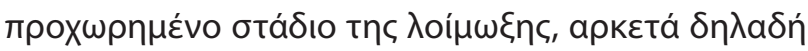

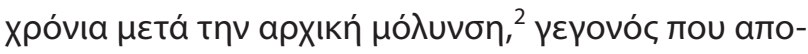

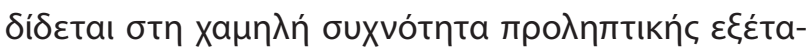

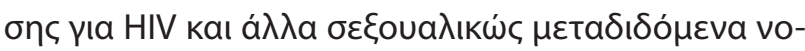

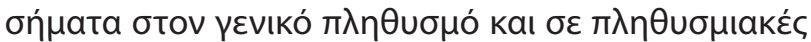

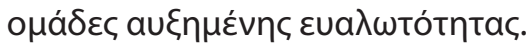

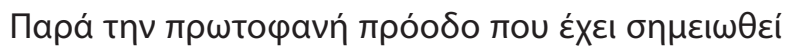

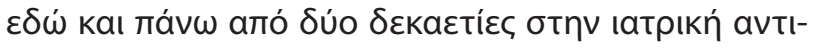

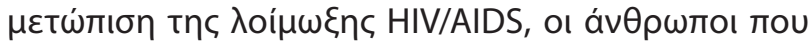

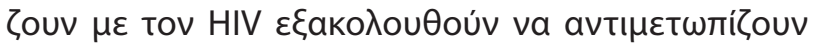

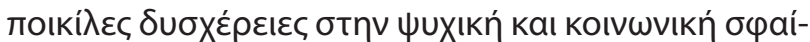

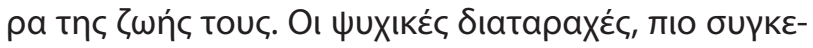

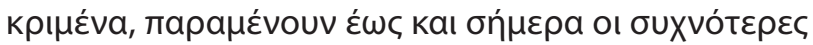

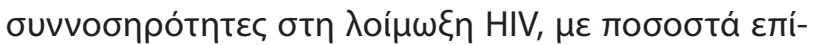

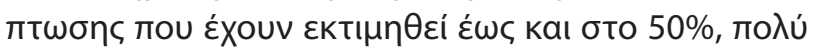

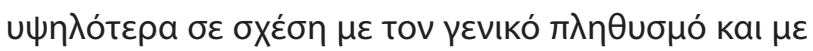

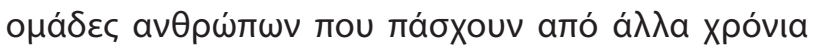

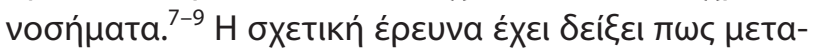

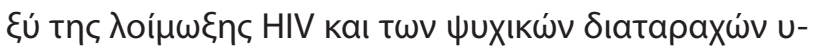

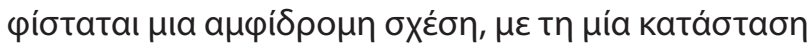

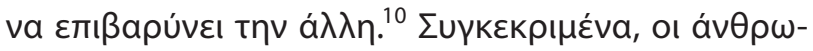

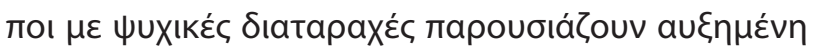

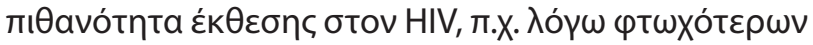

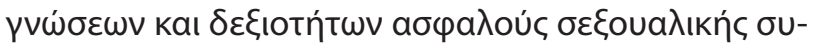

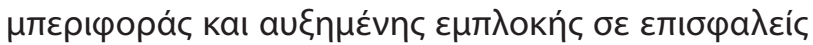

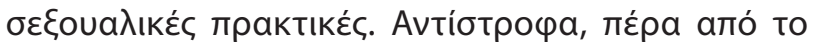

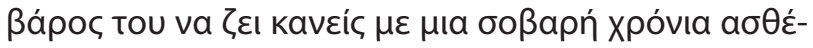

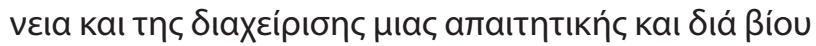

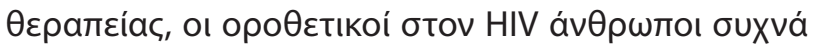

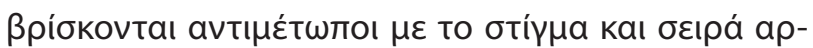

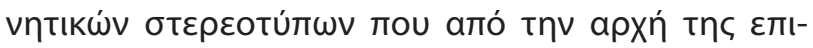

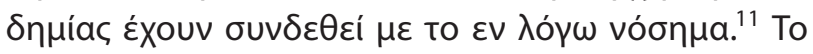

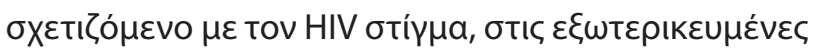

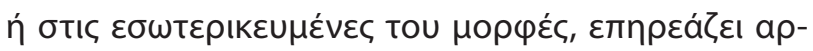

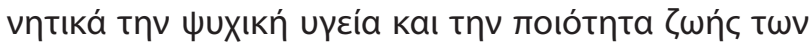

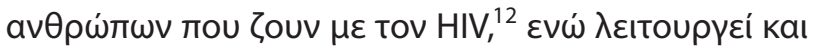

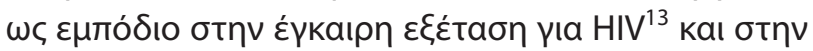

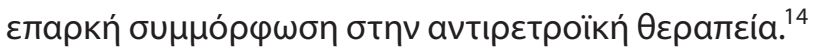




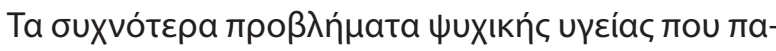

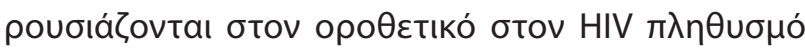

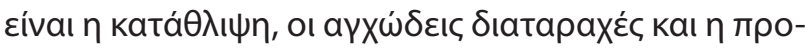

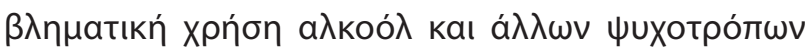

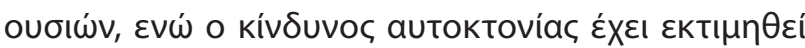

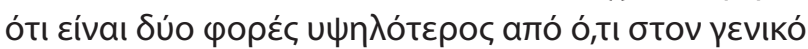

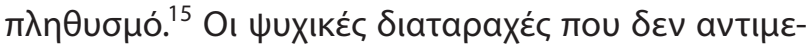

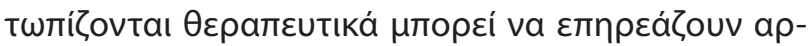

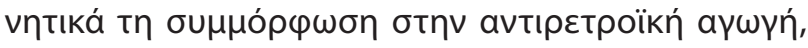

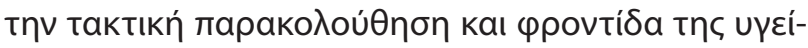

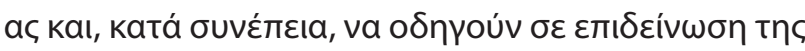

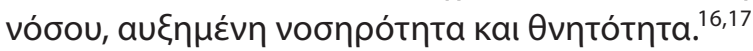

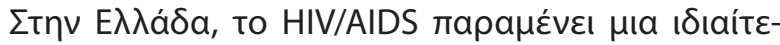

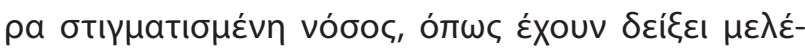

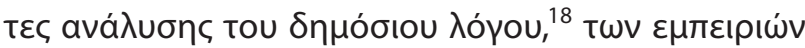

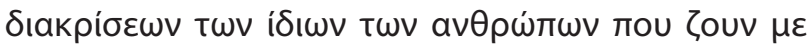

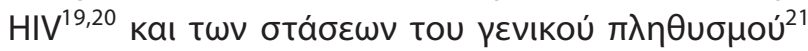

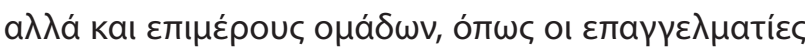

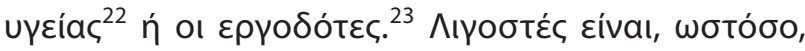

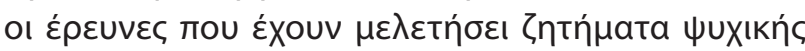

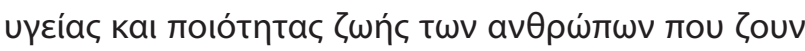

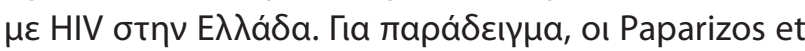

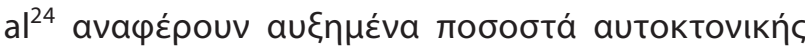

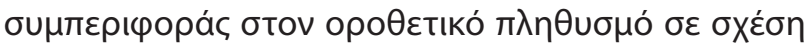

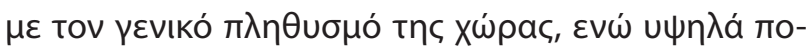

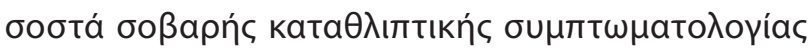

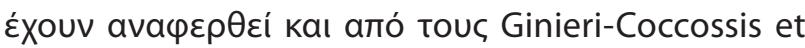

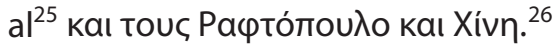

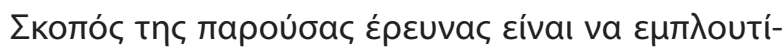

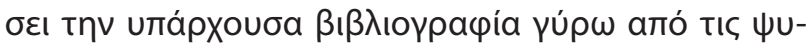

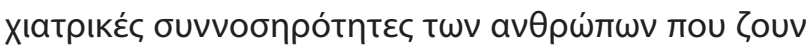

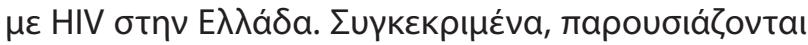

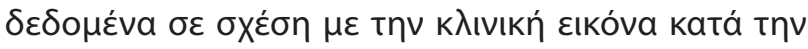

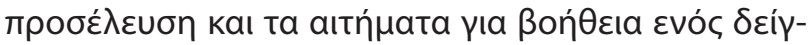

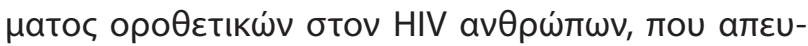

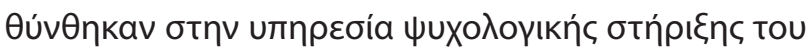

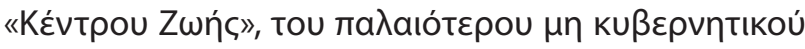

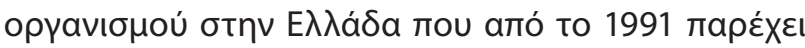

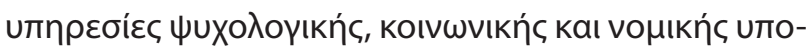

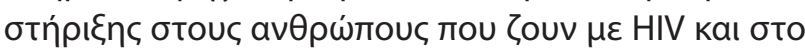

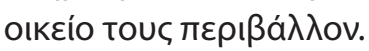

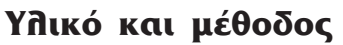

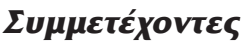

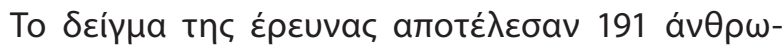

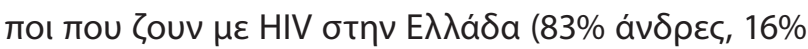

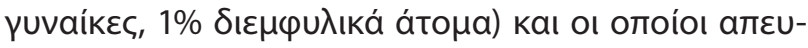

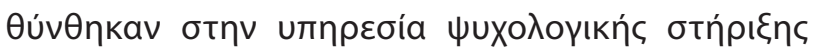

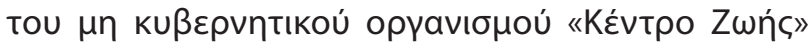

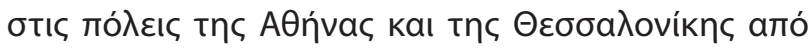

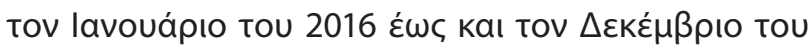

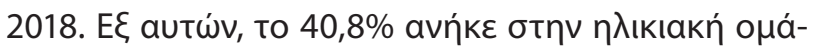

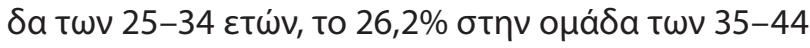

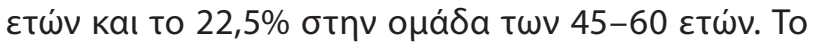

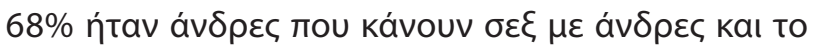
13,6\% ávӨ

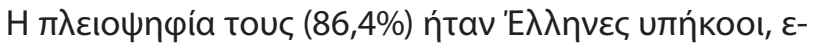

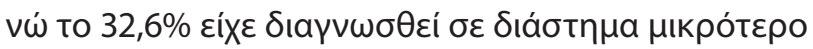

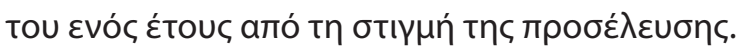

\section{Мıаঠıкабі́a}

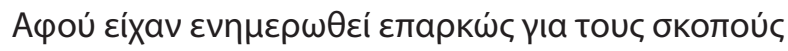

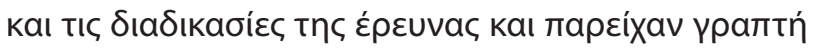

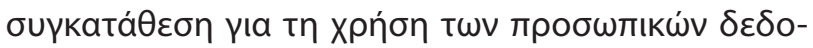

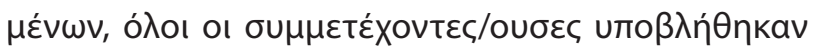

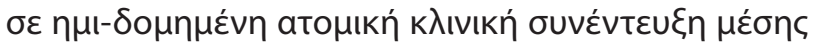

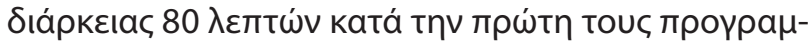

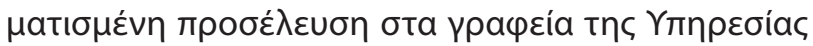

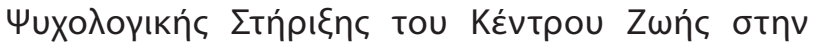

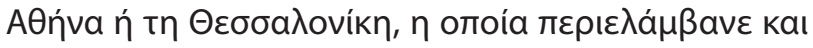

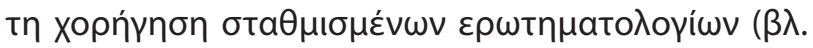

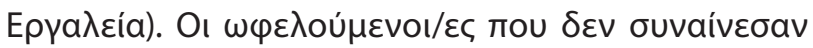

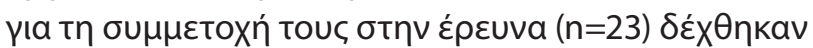

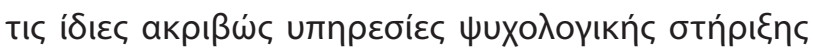

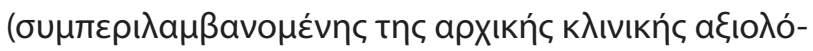

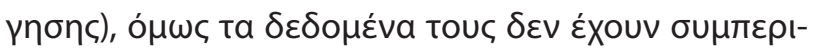

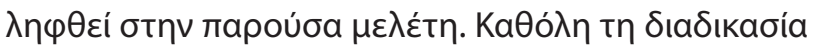

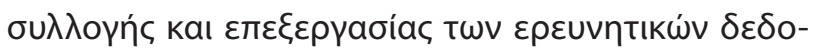

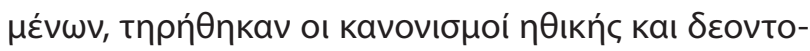

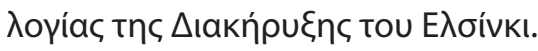

\section{Epyaneía}

Oı ато

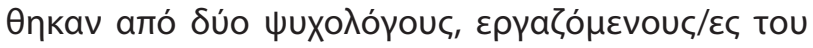

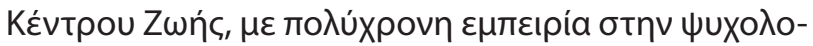

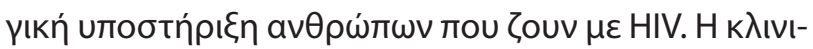

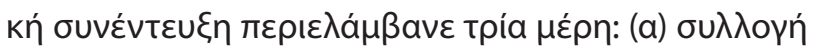

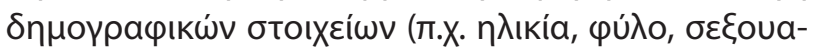

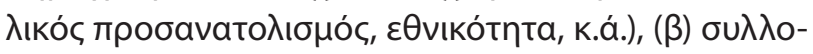

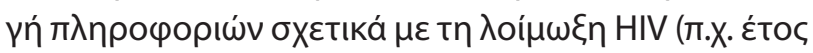

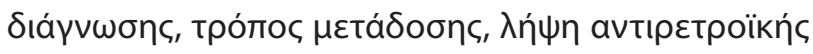

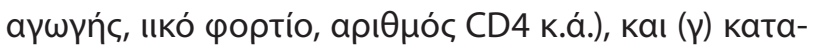

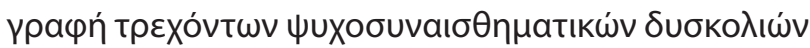

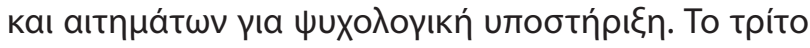

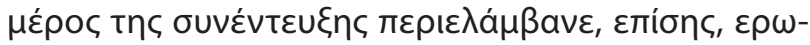




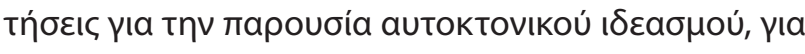

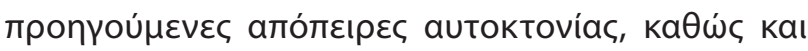

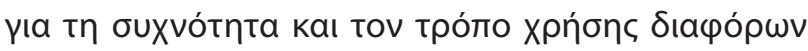

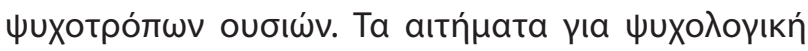

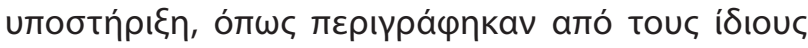

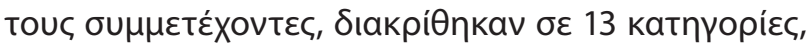

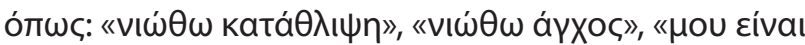

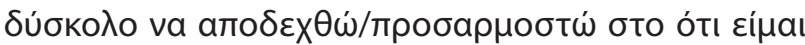

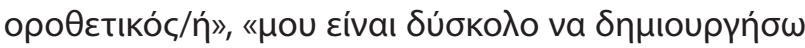

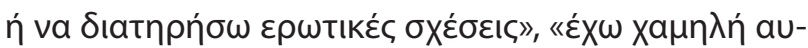

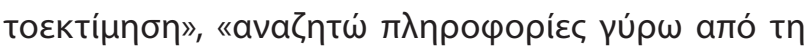

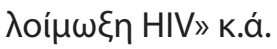

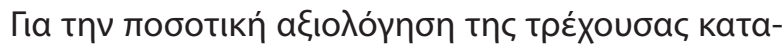

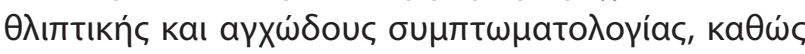

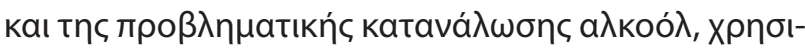

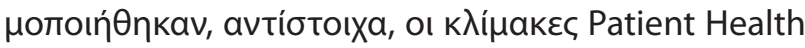
Questionnaire (PHQ-927,28), Beck Anxiety Inventory

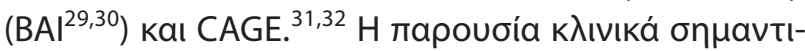

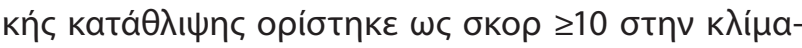

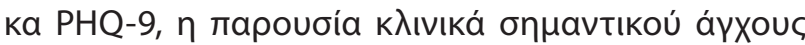

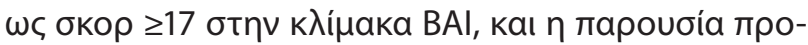

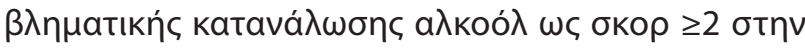

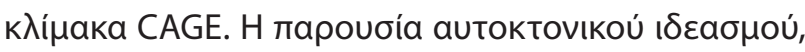

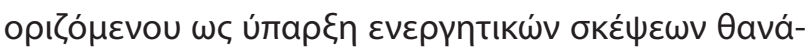

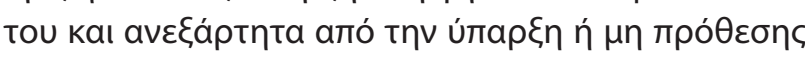

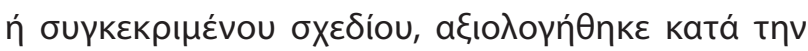

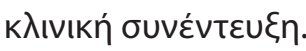

\section{Avánuø耳}

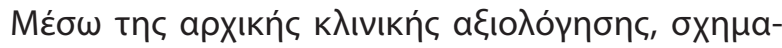

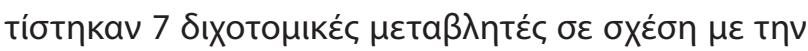

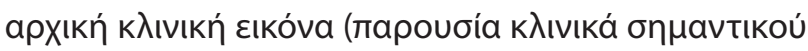

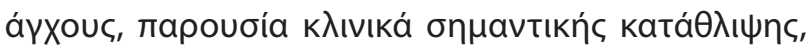

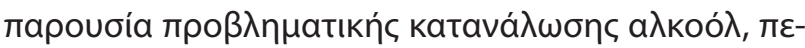

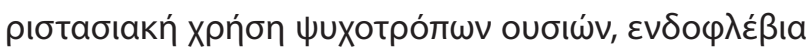

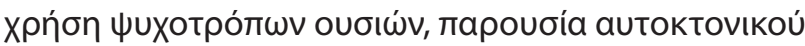

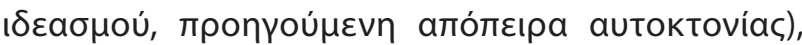

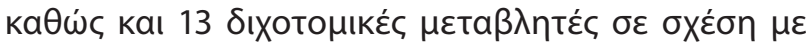

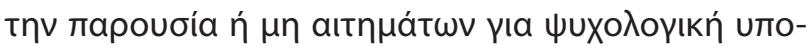

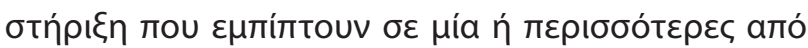

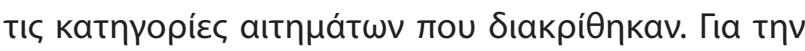

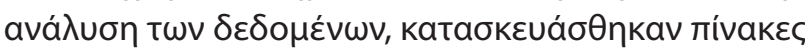

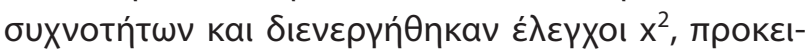

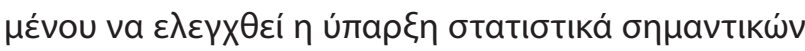

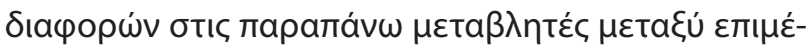

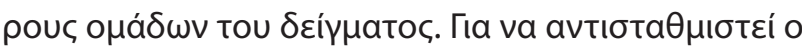

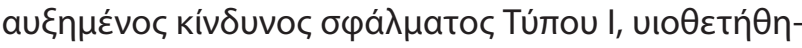

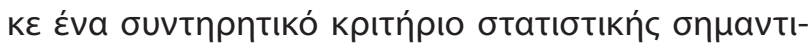

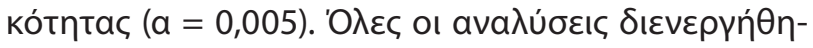

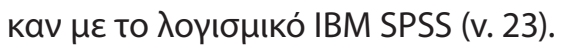

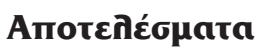

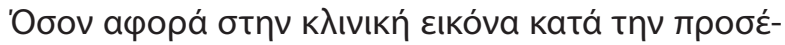

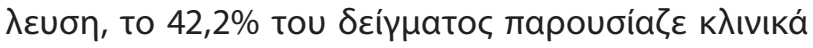

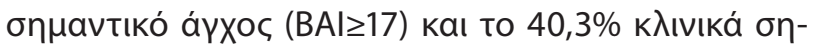

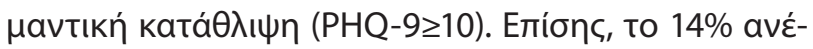

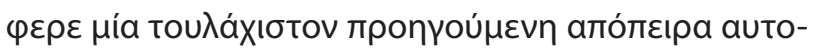

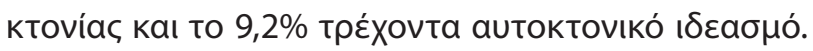

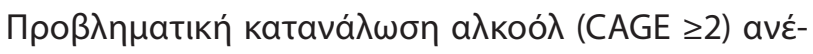

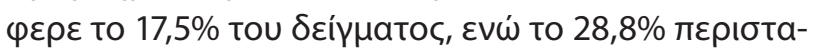

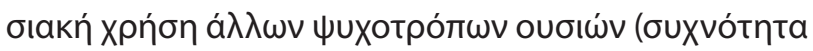

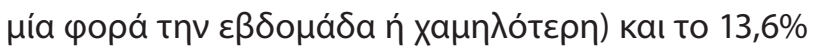

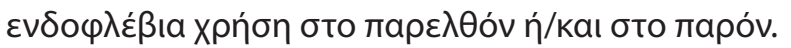

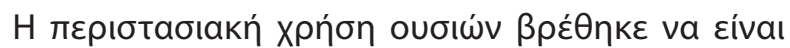

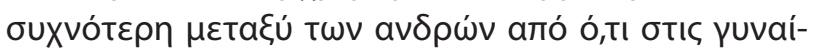

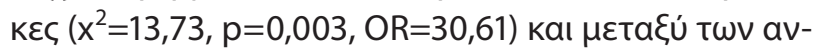

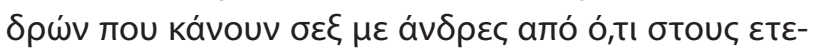

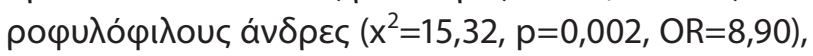

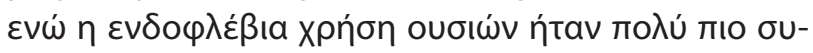

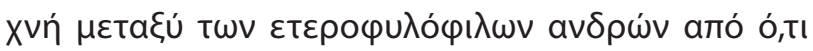

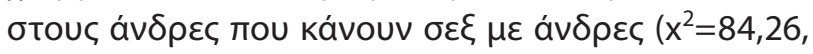

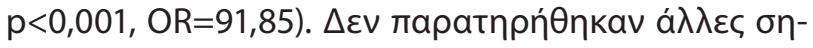

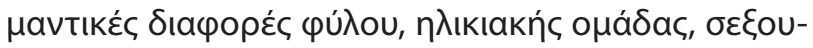

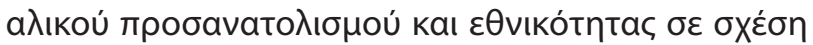

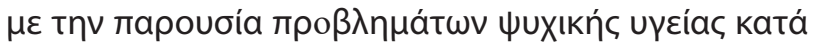

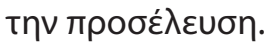

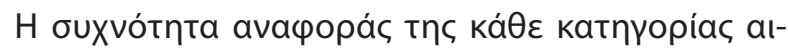

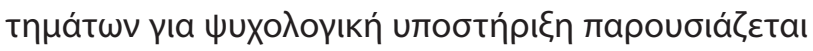

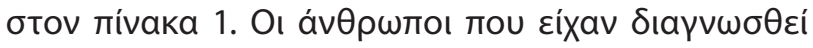

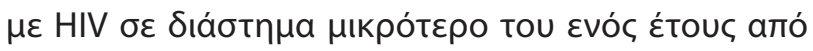

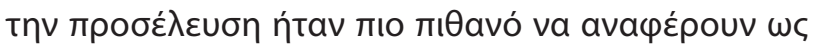

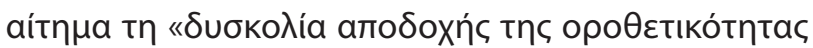

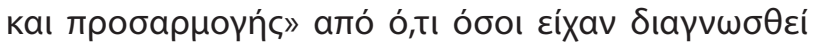

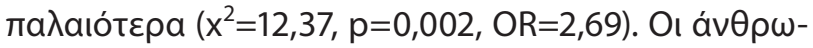

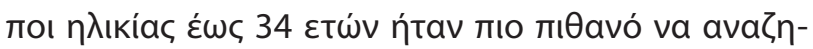

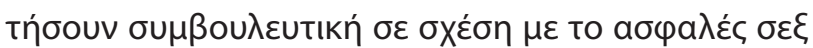

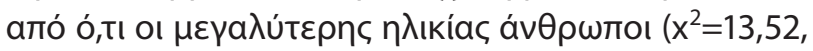

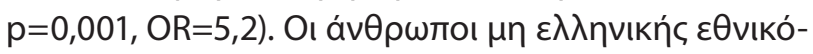

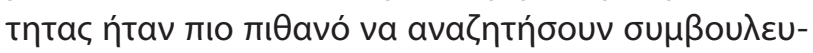

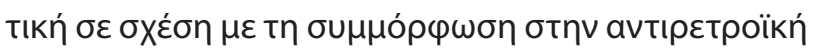

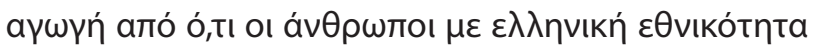

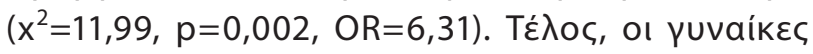

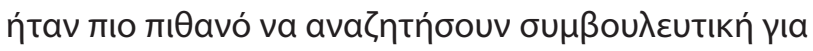

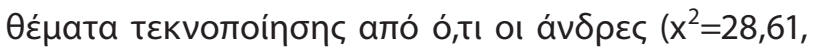




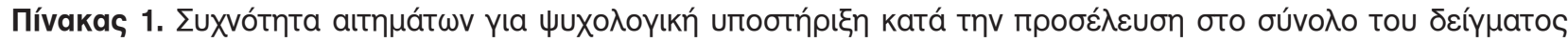
$(\mathrm{N}=191)$.

\begin{tabular}{|c|c|c|}
\hline 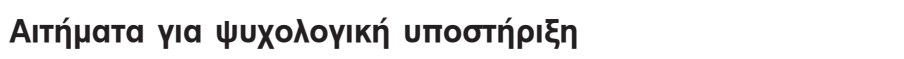 & $\mathbf{n}$ & (\%) \\
\hline 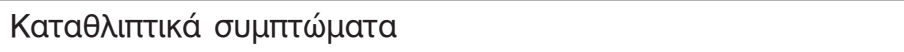 & 112 & $58,6 \%$ \\
\hline 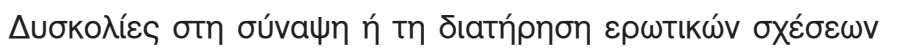 & 93 & $48,7 \%$ \\
\hline 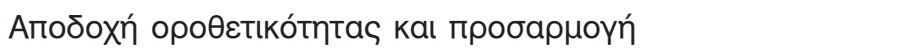 & 82 & $42,9 \%$ \\
\hline 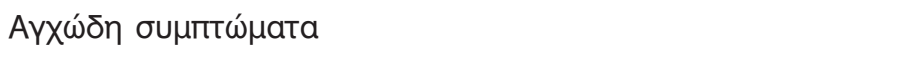 & 81 & $42,4 \%$ \\
\hline 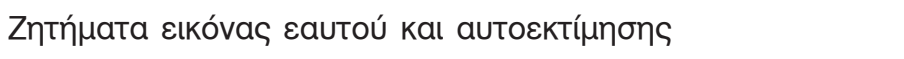 & 78 & $40,8 \%$ \\
\hline 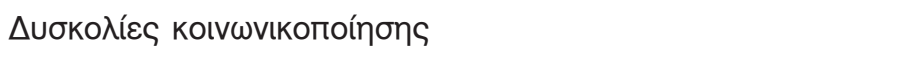 & 69 & $36,1 \%$ \\
\hline 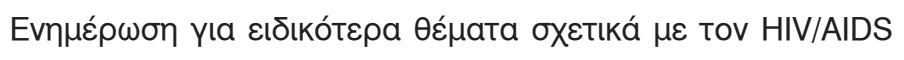 & 64 & $33,5 \%$ \\
\hline 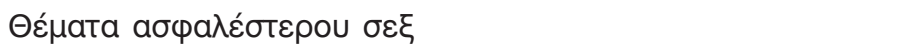 & 30 & $15,7 \%$ \\
\hline 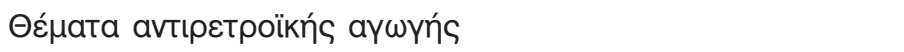 & 11 & $5,8 \%$ \\
\hline 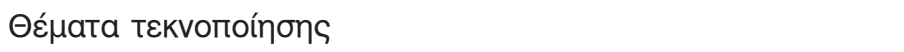 & 9 & $4,7 \%$ \\
\hline 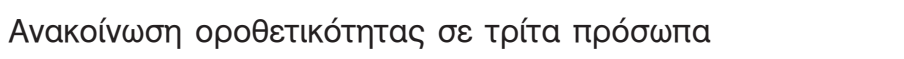 & 60 & $31,4 \%$ \\
\hline 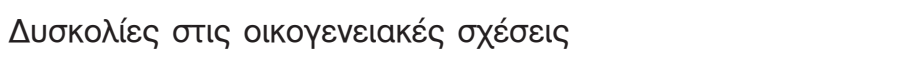 & 51 & $26,7 \%$ \\
\hline 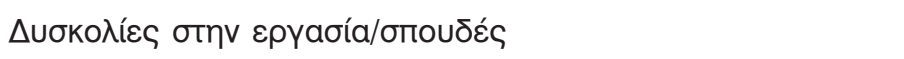 & 39 & $20,4 \%$ \\
\hline 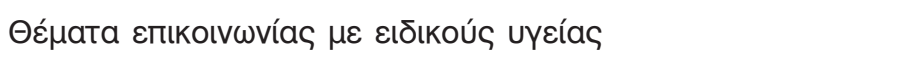 & 33 & $17,3 \%$ \\
\hline 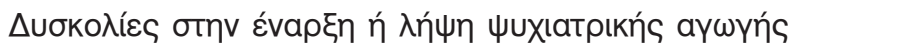 & 24 & $12,6 \%$ \\
\hline 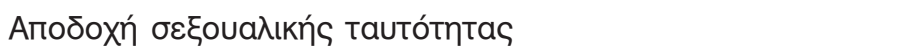 & 23 & $12,0 \%$ \\
\hline
\end{tabular}

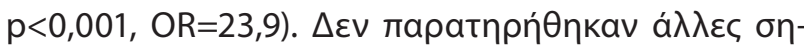

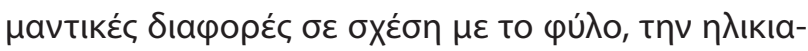

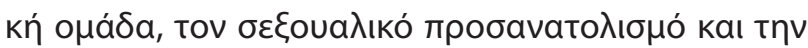

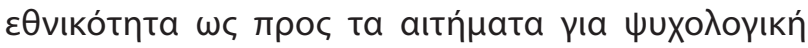

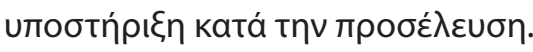

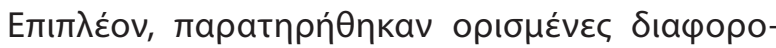

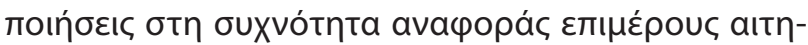

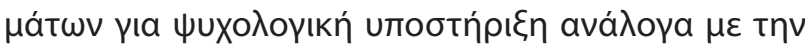

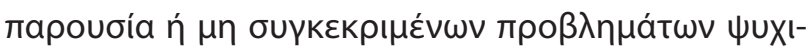

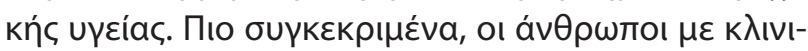

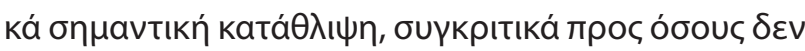

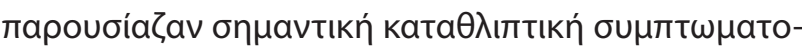

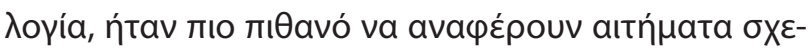

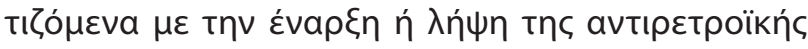

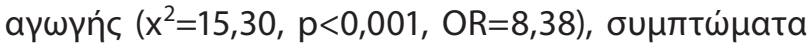

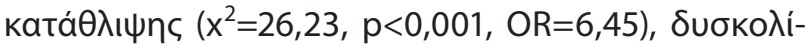

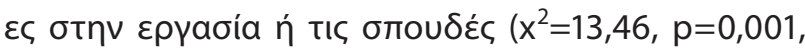

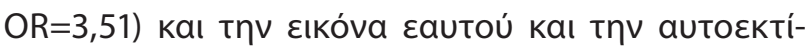

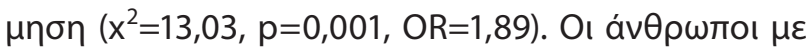

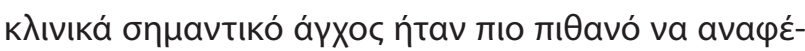

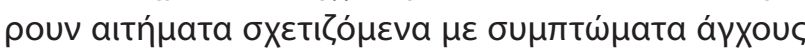
$\left(x^{2}=71,99, p<0,001, O R=19,43\right)$, $\varepsilon v \omega \dot{~ o l ~ a ́ v \theta \rho \omega \pi о ~} \mu \varepsilon$

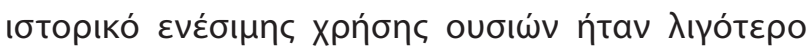

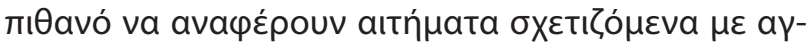

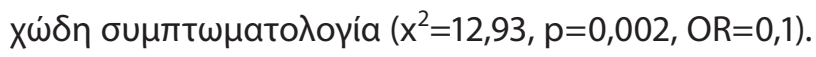

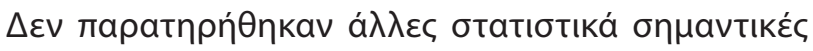

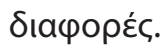

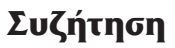

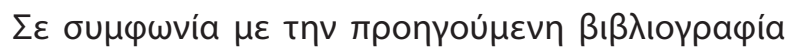

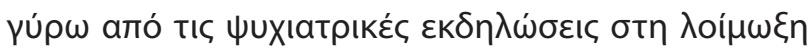

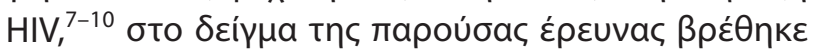

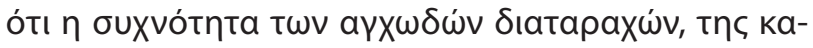

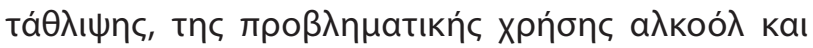

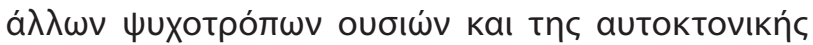

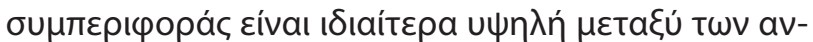

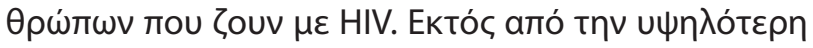

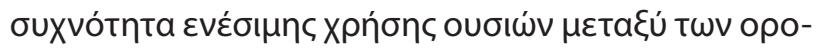

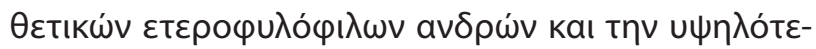

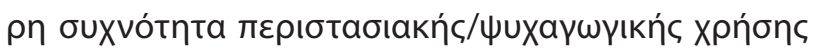

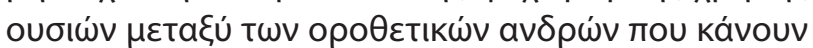

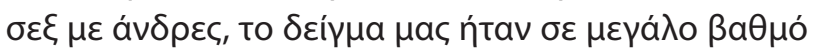

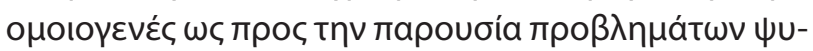

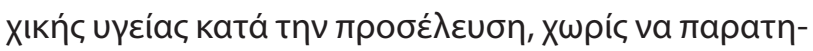

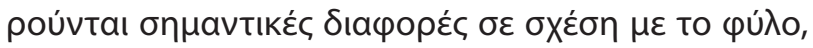

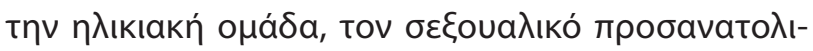

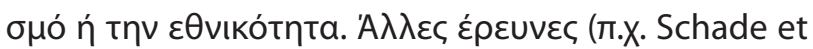

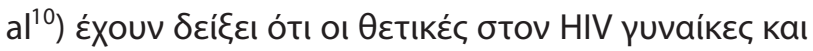




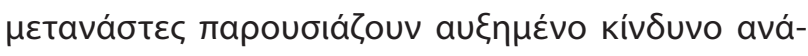

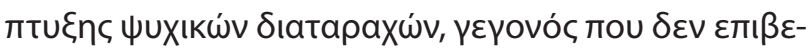

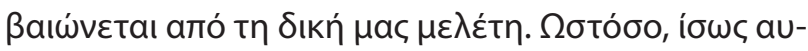

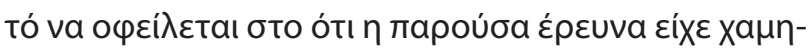

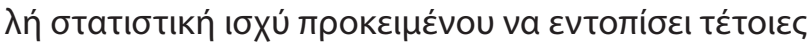

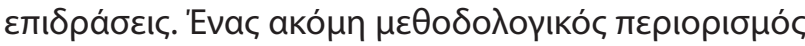

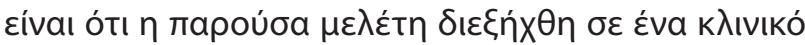

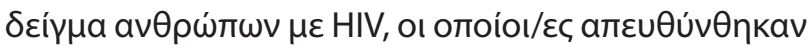

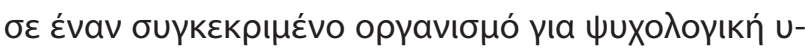

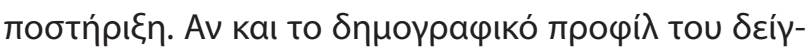

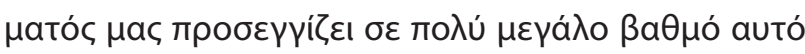

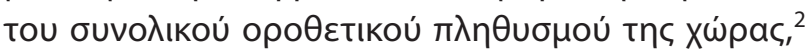

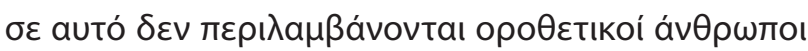

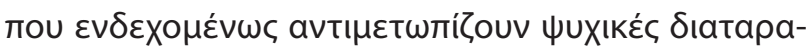

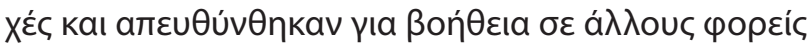

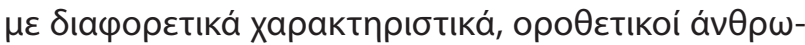

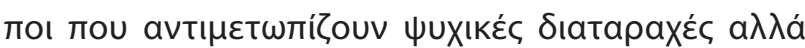

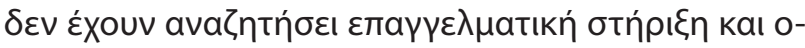

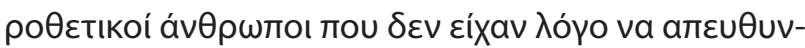

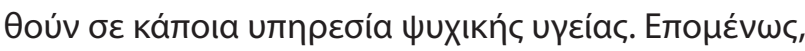

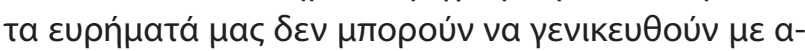

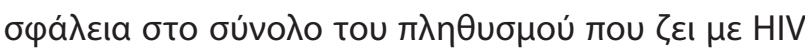
$\sigma \tau \eta v E \lambda \lambda a^{\prime} \delta a$.

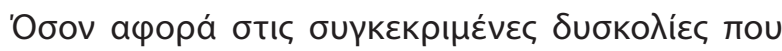

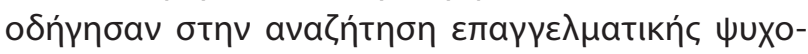

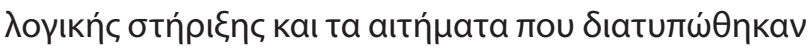

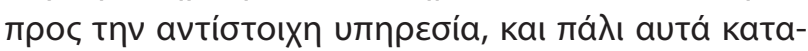

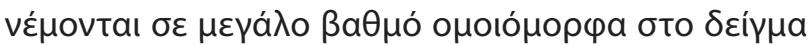

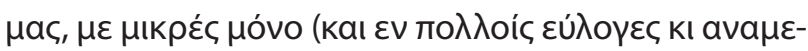

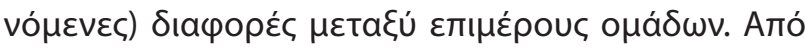

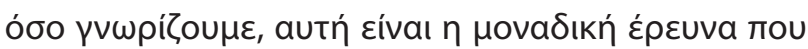

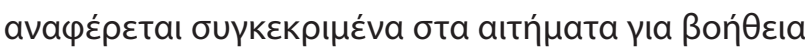

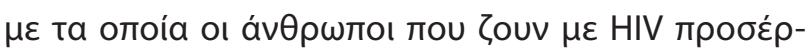

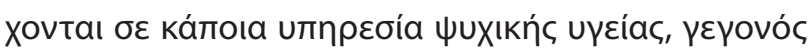

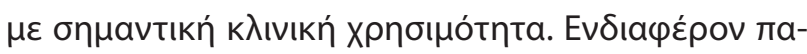

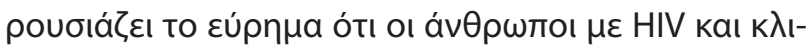

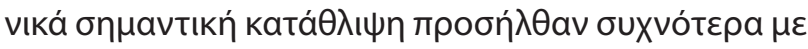

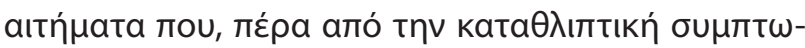

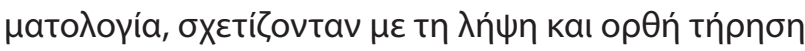

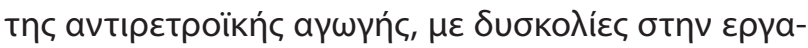

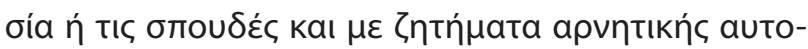

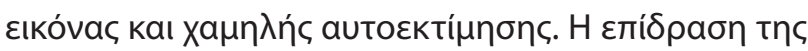

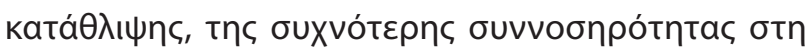

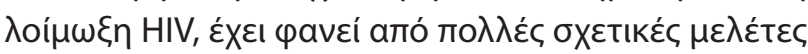

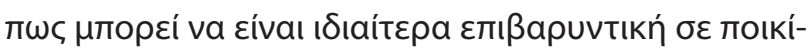

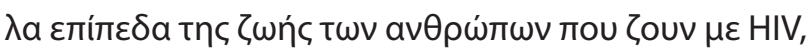

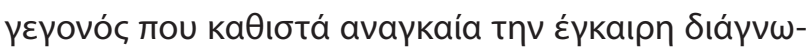

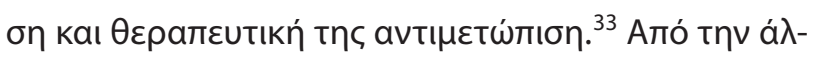

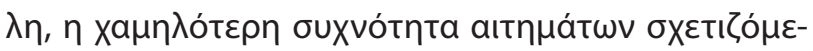

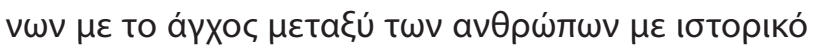

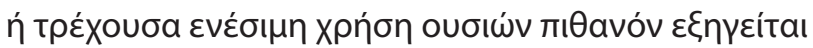

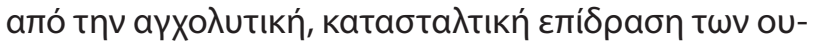

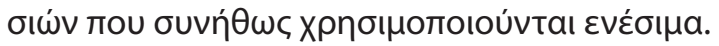

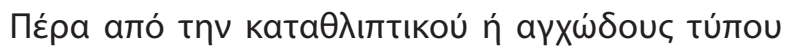

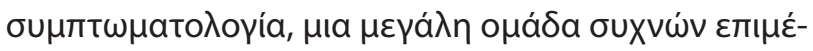

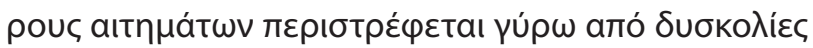

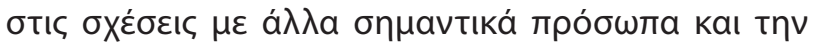

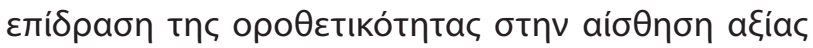

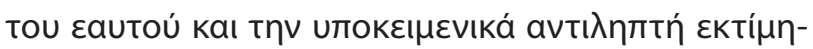

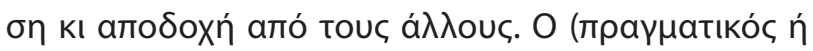

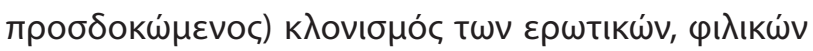

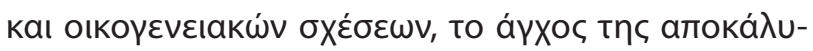

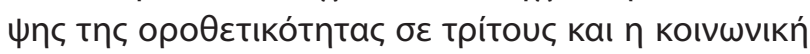

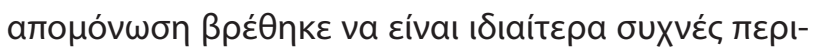

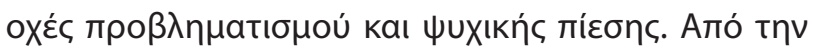

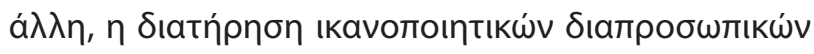

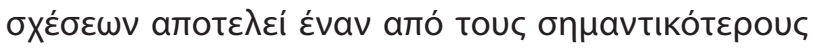

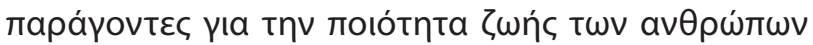

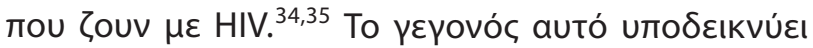

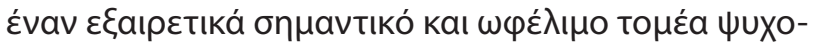

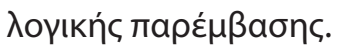

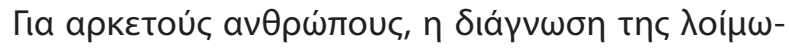

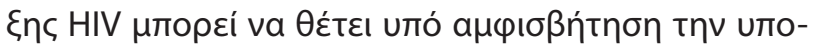

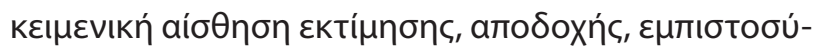

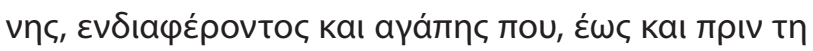

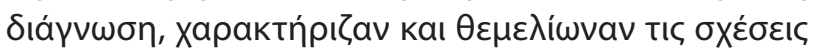

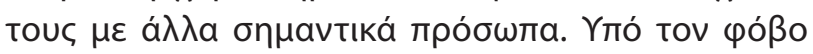

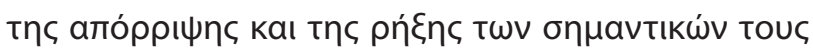

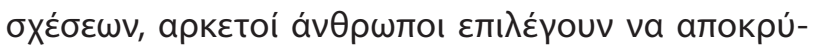

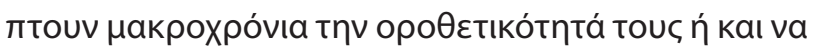

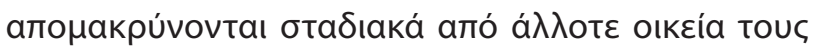

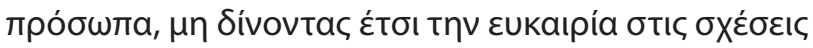

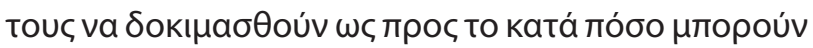

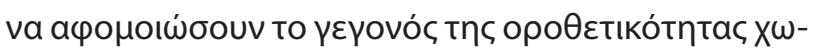

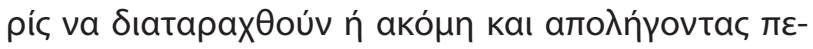

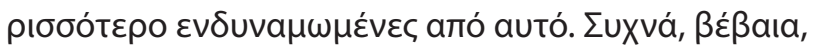

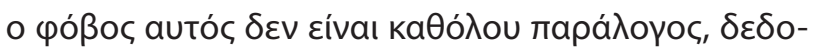

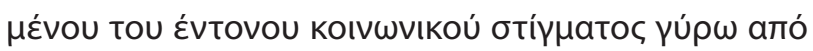

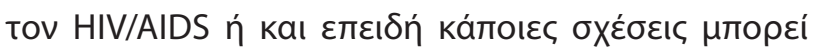

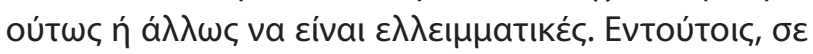

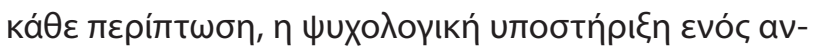

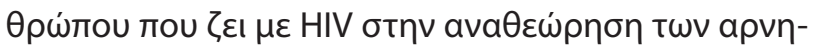

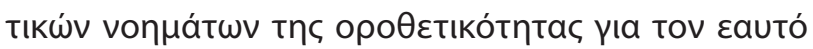

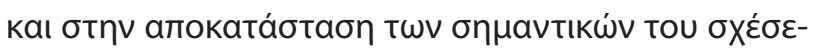




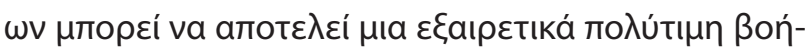

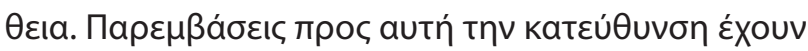

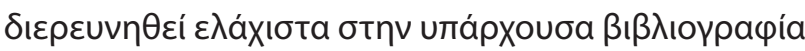

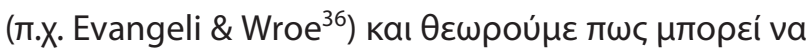

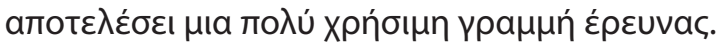

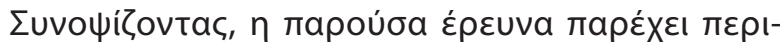

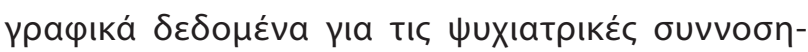

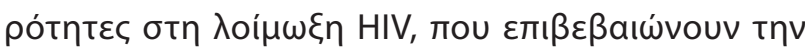

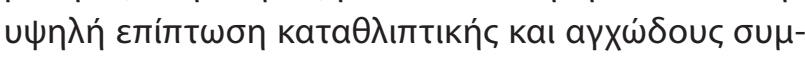

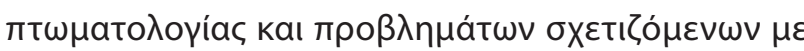

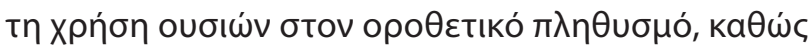

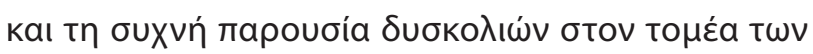

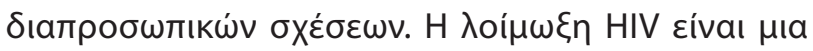

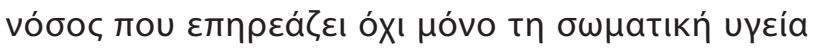

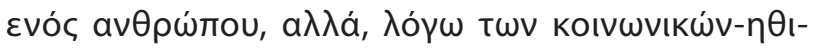

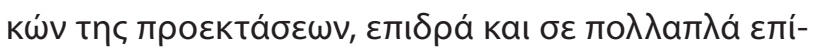

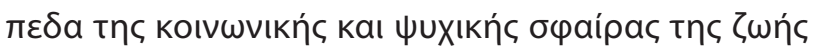

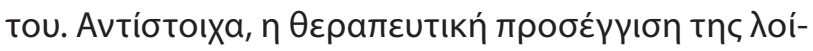

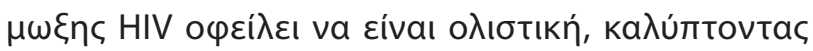

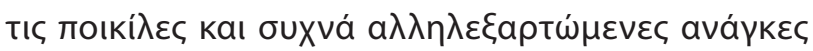

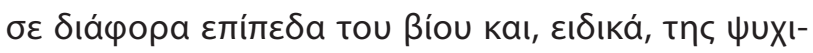
kńc uүعíac.

\title{
Clinical presentation and requests for support of people with HIV/AIDS seeking counselling and psychotherapy in Greece
}

\author{
A. Peltekis, ${ }^{1}$ M. Koulentianou ${ }^{2}$ \\ ${ }^{1}$ Centre for Life, Thessaloniki, ${ }^{2}$ Centre for Life, Athens, Greece
}

Psychiatriki 2019, 30:120-128

Despite the large progress during the last decades in the medical treatment of HIV/AIDS infection, people living with HIV nevertheless face multiple adversities at various levels of their lives. Mental disorders, in particular, are the most common comorbidities in HIV infection with negative consequences in adherence to antiretroviral medication, disease progress and overall quality of life. HIVrelated stigma, still quite intense in Greece, is one of the most debilitating factors concerning people's living with HIV mental health. The present study looked at the clinical presentation at intake and treatment requests of 191 (83\% males) people living with HIV who addressed the psychological support service of the non-governmental organization 'Centre for Life' during the years 201618. Data were collected through a semi-structured clinical interview and administration of adapted questionnaires (PHQ-9, BAI, CAGE), which resulted in 7 dichotomous variables related to clinical presentation and 13 dichotomous variables related to treatment requests at intake. To analyze data, we constructed frequency tables and performed chi-square tests. In the whole sample, $42.2 \%$ presented anxiety disorders, $40.3 \%$ depression, $28.8 \%$ occasional substance use, $17.5 \%$ problematic use of alcohol and $13.6 \%$ intravenous drug use. Moreover, $14 \%$ reported at least one suicide attempt in the past and 9.2\% current suicidal ideation. Apart from more frequent intravenous drug use among heterosexual males and more frequent occasional/recreational drug use among men who have sex with men, no other differences related to gender, age group, sexual orientation or ethnicity were observed in the initial clinical presentation. Similarly, the most frequent treatment requests were homogenously distributed in our sample, such as depressive symptoms (58.6\%), difficulties in romantic relationships (48.7\%), accepting being HIV positive (42.9\%), anxiety symptoms (42.4\%) and issues of negative self-esteem (40.8\%). The presence of clinically significant depression was found to be related to a wider range of treatment requests compared to other mental health problems. A large group 
of requests focused on interpersonal relationships difficulties (e.g. disclosure anxiety, social isolation, disturbed relationships with familiar persons). This indicates an important area of psychological intervention. HIV infection may affect many levels of an individual's life, including their mental health. Respectively, HIV treatment needs to adopt a more holistic approach.

Key words: HIV/AIDS infection, mental health problems, requests for psychological support, interpersonal relationships.

\section{Bıßntoypacpía}

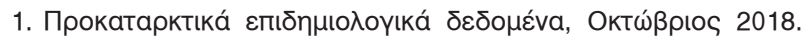

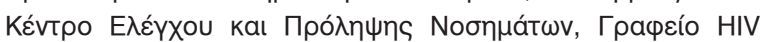

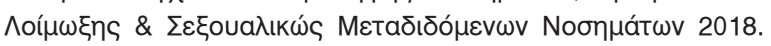

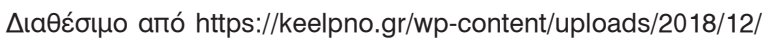
epidimiologiko-31-10-2018_final.pdf

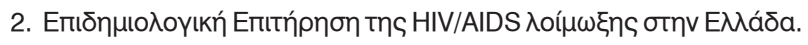

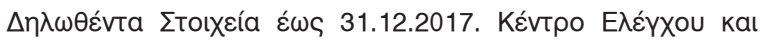

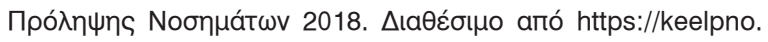
gr/wp-content/uploads/2018/12/epidimiologiko-2017.pdf

3. The Antiretroviral Cohort Collaboration. Survival of HIV-positive patients starting antiretroviral therapy between 1996 and 2013: a collaborative analysis of cohort studies. Lancet HIV 2017, 4:e349-e356, doi: 10.1016/S2352-3018(17)30066-8

4. Rodger A, Cambiano V, Bruun T, Vernazza P, Collins S, Corbelli GM et al. Risk of HIV transmission through condomless sex in MSM couples with suppressive ART: The PARTNER2 Study extended results in gay men. Профорıки́ пароuбíaon бт 220

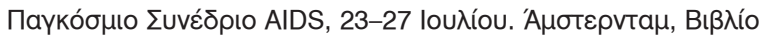

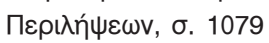

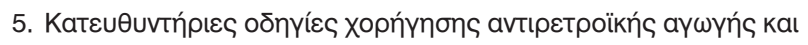

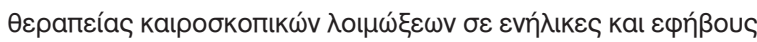

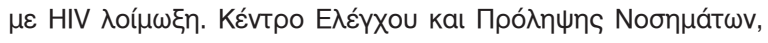

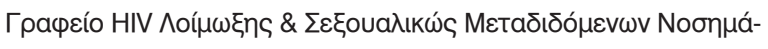

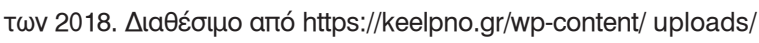
2018/12/antiretroiki-agogi-11-05-2018.pdf

6. Vourli G, Nikolopoulos G, Paparizos V, Skoutelis A, Metallidis S, Gargalianos P et al. HIV cascade of care in Greece: useful insights from additional stages. PLoS One 2018, 13: e0207355, doi: 10.1371/journal.pone.0207355

7. Adams C, Zacharia S, Masters L, Coffey C, Catalan P. Mental health problems in people living with HIV: changes in the last two decades: the London experience 1990-2014. AIDS Care 2016, 28:56-59, doi: 0.1080/09540121.2016.1146211

8. Kendall CE, Wong J, Taljaard M, Glazier RH, Hogg W, Younger $\mathrm{J}$ et al. A cross-sectional, population-based study measuring comorbidity among people living with HIV in Ontario. BMC Public Health 2014, 14:161, doi: 10.1186/1471-2458-14-161

9. Edmiston N, Passmore E, Smith DJ, Petoumenos K. Multimorbidity among people with HIV in regional New South Wales, Australia. Sex Health 2015, 12:425-432, doi: 10.1071/SH14070

10. Schadı A, van Grootheest G, Smit JH. HIV-infected mental health patients: characteristics and comparison with HIV-infected patients from the general population and non-infected mental health patients. BMC Psychiatry 2013, 13:35, doi: 10.1186/1471244X-13-35
11. Holzemer WL, Human S, Arudo J, Rosa ME, Hamilton MJ, Corless I et al. Exploring HIV stigma and quality of life for persons living with HIV infection. J Assoc Nurses AIDS Care 2009, 20:161-168, doi: 10.1016/j.jana.2009.02.002

12. Fuster-Ruizdeapodaca MJ, Molero F, Holgado FP, Mayordomo S. Enacted and internalized stigma and quality of life among people with HIV: the role of group identity. Qual Life Res 2014, 23:1967-1975, doi: 10.1007/s11136-014-0653-4

13. Gesesew HA, Gebremedhin AT, Demissie TD, Kerie MW, Sudhakar M, Mwanri L. Significant association between perceived HIV related stigma and late presentation for HIV/AIDS care in low and middle-income countries: A systematic review and meta-analysis. Qual Life Res 2014, 23:1967-1975, doi: 10.1371/journal.pone. 0173928

14. Katz IT, Ryu AE, Onuegbu AG, Psaros C, Weiser SD, Bangsberg DR et al. Impact of HIV-related stigma on treatment adherence: systematic review and meta-synthesis. J Int AIDS Soc 2013, 16:18640, doi: 10.7448/IAS.16.3.18640

15. Jallow A, Ljunggren $G$, Wändell $P$, Wahlström L, Carlsson AC. HIV-infection and psychiatric illnesses - A double edged sword that threatens the vision of a contained epidemic: The Greater Stockholm HIV Cohort Study. J Infect 2016, 74:22-28, doi: 10.1016/j.jinf.2016.09.009

16. Rueda S, Mitra S, Chen S, Gogolishvili D, Globerman J, Chambers $L$ et al. Examining the associations between HIVrelated stigma and health outcomes in people living with HIV/ AIDS: a series of meta-analyses. BMJ Open 2016, 6:e011453, doi: 10.1136/bmjopen-2016-011453

17. Vagenas P, Azar MM, Copenhaver MM, Springer SA, Molina $\mathrm{PE}$, Altice FL. The impact of alcohol use and related disorders on the HIV continuum of care: a systematic review. Curr HIV/ AIDS Rep 2015, 12:421-436, doi: 10.1007/s11904-015-0285-5

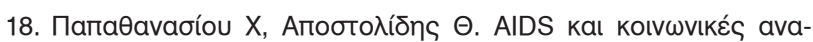

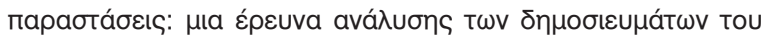

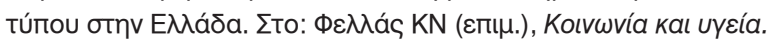

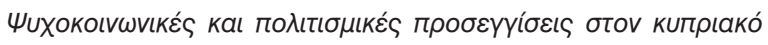

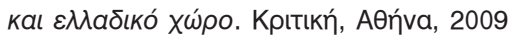

19. Kalemi G, Gkioka S, Tsapatsari P, Tzeferakos G, Kandri T, Psarra ML. Stigma and self-esteem: a case of HIV-positive sex-workers. Psychiatriki 2017, 28:67-74, doi: 10.22365/jpsych.2017.281.67

20. The Greek Association of PLHIV - Positive Voice. The people

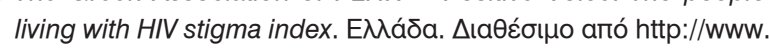
stigmaindex.org/sites/default/files/reports/Stigmalndex_Greece_ Final_ENG.pdf 


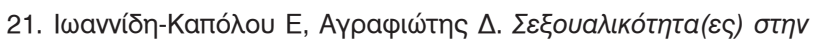

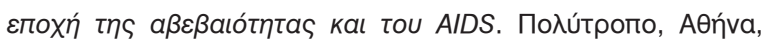
2005

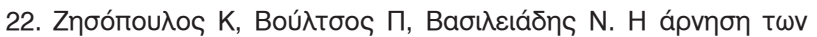

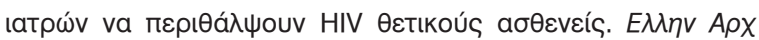
AIDS 2010, 18:54-60

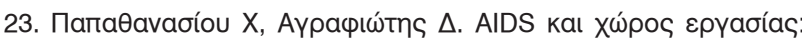

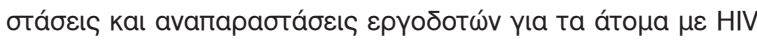

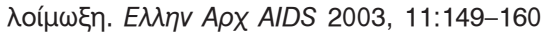

24. Paparizos V, Triantafyllopoulou I, Kourkounti S, Retsas T, Paparizou E. Suicidal behaviour in HIV-infected patients in Greece. Infez Med 2017, 25:64-70, PMID: 28353458

25. Ginieri-Coccossis M, Triantafillou E, Papanikolaou N, Baker R, Antoniou C, Skevington SM et al. Quality of life and depression in chronic sexually transmitted infections in UK and Greece: the use of WHOQOL-HIV/STI BREF. Psychiatriki 2018, 29:209-219, doi: $10.22365 /$ jpsych.2018.293.209

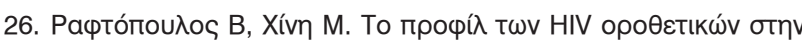

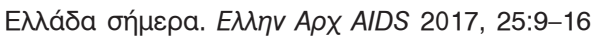

27. Kroenke K, Spitzer RL, Williams JB. The PHQ-9: validity of a brief depression severity measure. J Gen Intern Med 2001, 16:606613, PMID: 11556941

28. Karekla M, Pilipenko N, Feldman J. Patient Health Questionnaire: Greek language validation and subscale factor structure. Compr Psychiatry 2012, 53:1217-1226, doi: 10.1016/j.comppsych.2012.05.008

29. Beck AT, Epstein N, Brown G, Steer RA. An inventory for measuring clinical anxiety: psychometric properties. J Consult Clin Psychol 1988, 56:893-897, doi: 10.1037/0022-006X.56.6.893

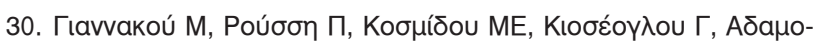

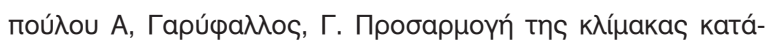

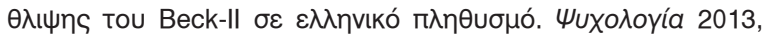
$10: 120-146$
31. Ewing JA. Detecting alcoholism. The CAGE questionnaire. JAMA 1984, 252:1905-1907, PMID: 6471323

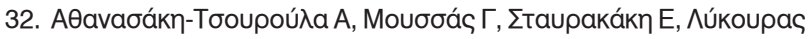

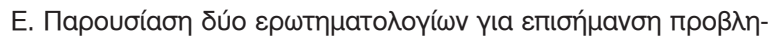

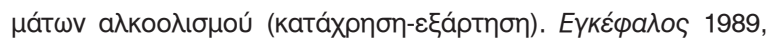
26:71-74

33. Nanni MG, Caruso R, Mitchell AJ, Meggiolaro E, Grassi L. Depression in HIV infected patients: a review. Curr Psychiatry Rep 2015, 17:530, doi: 10.1007/s11920-014-0530-4

34. Degroote S, Vogelaers D, Vandijck DM. What determines health-related quality of life among people living with HIV: an updated review of the literature. Arch Publ Health 2014, 72:40, doi: 10.1186/2049-3258-72-40

35. Drewes J, Gusy B, Róden U. More than 20 years of research into the quality of life of people with HIV and AIDS: a descriptive review of study characteristics and methodological approaches of published empirical studies. J Int Assoc Provid AIDS Care 2013, 12:18-22, doi: 10.1177/1545109712456429

36. Evangeli M, Wroe AL. HIV disclosure anxiety a systematic review and theoretical synthesis. AIDS Behav 2017, 21:1-11, doi: 10.1007/ s10461-016-1453-3

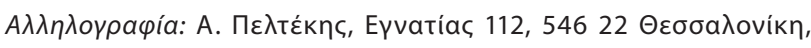
$\operatorname{T\eta } \lambda$ : 2310-23 70 40, e-mail: a.peltekis@kentrozois.gr 